C. DAI AND L. JIN

KODAI MATH. J.

10 (1987). $74-82$

\title{
NUMBER OF DEFICIENT VALUES OF A CLASS of MEROMORPHIC FUNCTION
}

\author{
By Chongui DAI AND LU JiN
}

\begin{abstract}
We proved the following Theorem

Theorem. Let $f(z)$ be a meromorphic function of lower order $\mu<\infty$. If $\sum_{a} \delta\left(a, f^{\prime}\right)=2$ then we have

$$
P_{0}+P_{1} \leqq \mu+1,
$$

where $P_{0}, P_{1}$ are the numbers of finite deficient values of $f(z), f^{\prime}(z)$ respec. tively.
\end{abstract}

\section{Lemmas.}

We need the following four known results.

LEMMA A [1 Theorem 1]. Let $f(z)$ be a meromorphic function of lower order $\mu<\infty$. Assume that there exists a positive integer $P$ which satifies

$$
P-\frac{1}{2} \leqq \mu<P+\frac{1}{2} \text {. }
$$

Assume also that for some $A_{0}>0$ and $0<\varepsilon<1$,

then

$$
K(f)=\varlimsup_{r \rightarrow \infty} \frac{N(r, f)+N(r, 1 / f)}{T(r, f)}<\frac{\varepsilon}{A_{0}(P+1)},
$$

1) $P \geqq 1$.

2) For $r>r_{0}$ and all $1<\sigma \leqq 36$, we have

$$
\left\{\begin{array}{l}
T(\sigma r, f)=\sigma^{P} T(r, f)(1+\eta(r, \sigma)) \\
|\eta(r, \sigma)|<\varepsilon .
\end{array}\right.
$$

3) Let $E(\mu, P)$ denotes the Weierstrass primary factor of genus $P$ and $a_{\nu}, b_{\mu}$ $(\nu=1,2, \cdots ; \mu=1,2, \cdots)$ are zeros and poles of $f(z)$ respectively, then we have

Received June 6, 1986 


$$
f(z)=z^{k} e^{\alpha_{0} z^{P}+\alpha_{1} z^{P-1}+\cdots+\alpha_{P}} \frac{\prod_{\nu=1}^{\infty} E\left(\frac{z}{a_{\nu}}, P\right)}{\prod_{\mu=1}^{\infty} E\left(\frac{z}{b_{\mu}}, P\right)},
$$

where $k$ is an integer. If we set

$$
C(r)=\alpha_{0}+\frac{1}{P}\left\{\sum_{\left|a_{\nu}\right| \leqq 1} a_{\nu}^{-P}-\sum_{\left|b_{\mu}\right| \leqq r} b_{\mu}^{-P}\right\},
$$

then for $r>r_{0}$

$$
\left\{\begin{array}{l}
|C(\sigma r)-C(r)|<\varepsilon|C(r)| \\
T(r, f)=\left(1+\eta_{1}(r)\right) \frac{|C(r)|}{\pi} r^{P}, \quad\left|\eta_{1}(r)\right|<\varepsilon .
\end{array}\right.
$$

LEMMA B [1 Lemma 5]. Let the assumptions and notations be the same as in Lemma $A$, we further assume that

$$
K(f)<\frac{\varepsilon}{B_{0}(P+1) \log (P+1)+B_{1}(P+1) \log 1 / \delta},
$$

where $0<\varepsilon \leqq 1, o<\delta<1 / e$, and $B_{1}, B_{0}, A_{0}$ are constants which satisfy $B_{1}>B_{0}>A_{0}$, set $\alpha=\exp \left(\frac{1}{P+1}\right)$ and $C_{\jmath}=C\left(\alpha^{j}\right)$, where $j$ is an integer satifying $\alpha^{j} \leqq r<\alpha^{j+1}$, then for $z \in \Gamma_{j}-E_{j}(|z|=r), j \geqq j_{0}$,

$$
|\log | f(z)\left|-\operatorname{Re}\left\{C_{j} z^{P}\right\}\right|<4 \varepsilon\left|C_{j}\right| r^{P},
$$

where $\left.\Gamma_{j}=z, \alpha^{j}<|z| \leqq \alpha^{j+(3 / 2)}\right\}, E$, is the set of a finite number of circles which contain the zeros and poles of $f(z)$ and the sum of radius of those circles is no more than $4 e \delta \alpha^{j+2}$.

LEMMA C [4 Theorem 4.1]. Let $f(z)$ be a meromorphic fuction. Then for $\gamma>\gamma_{0}$

$$
T(r, f)<C\left\{T\left(2 r, f^{\prime}\right)+\log r\right\},
$$

where $C$ is a constant which is only dependent on $f(0)$.

LEMMA D [3, Proof of Theorem]. Let assumptions be the same as the Theorem, then

$$
K\left(f^{\prime \prime}\right)=\varlimsup_{r \rightarrow \infty} \frac{N\left(r, f^{\prime \prime}\right)+N\left(r, 1 / f^{\prime \prime}\right)}{T\left(r, f^{\prime \prime}\right)}=0 .
$$

\section{Main Lemmas.}

LEMMA 1. Let the assumptions be the same as the Theorem, then for an arbitrary set of finite deficient values $\left\{a_{1}, a_{2}, \cdots, a_{\tau}\right\}$ and $\left\{a_{\tau+1}, \cdots a_{\tau^{\prime}}\right\}$ of $f(z)$ and $f^{\prime}(z)$, there exists a set of positive numbers $\left\{\rho_{j}\right\}_{j=1}^{\infty}, \quad \alpha^{j} \leqq \rho_{j}<\alpha^{j+1}$ and sets $\left\{E_{1 \jmath}, E_{2 \jmath}, \cdots, E_{\tau \jmath}, \cdots, E_{\tau^{\prime} j}\right\}$ which are on $|z|=\rho$, so that meas $E_{l=}^{*}=\operatorname{meas}\{\phi ; 0$ 
$\left.\leqq \phi<2 \pi, \rho_{j} e^{\imath \phi} \in E_{l j}\right\}>\eta>0\left(l=1,2, \cdots, \tau^{\prime}\right)$, and for $z \in E_{l}$, we have

and

$$
\begin{array}{ll}
\log \left|f(z)-a_{l}\right|<-k T\left(\rho_{\jmath}, f^{\prime \prime}\right), & l=1,2, \cdots, \tau . \\
\log \left|f^{\prime}(z)-a_{l}\right|<-k T\left(\rho_{\jmath}, f^{\prime \prime}\right), & l=\tau+1, \tau+2, \cdots, \tau^{\prime} .
\end{array}
$$

$$
\begin{array}{ll}
\log \left|f^{\prime}(z)\right|<-k T\left(\rho_{\jmath}, f^{\prime \prime}\right), & l=1,2, \cdots, \tau . \\
\log \left|f^{\prime \prime}(z)\right|<-k T\left(\rho_{\jmath}, f^{\prime \prime}\right), & l=1,2, \cdots, \tau^{\prime} .
\end{array}
$$

where $\alpha=\exp (1 / \mu+1), k, \eta$ are absolute constants.

In order to prove Lemma 1, we must prove following Lemma.

LEMMA 2. Let $g(z)$ be meromorphic in $|z|<R(0<R<\infty)$, then for $z=r e^{i \theta}$ and $\rho, r<\rho \leqq R$,

$$
\begin{aligned}
\log ^{+} \frac{\left|g^{\prime}(z)\right|}{|g(z)|} \leqq & 5 \log 2+\log ^{+} \rho+3 \log ^{+} \frac{1}{\rho-r}+\log ^{+} \Re+\log ^{+} \frac{1}{\delta(z)} \\
& +\log ^{+} \frac{1}{\gamma}+\log ^{+} T(\rho, g)+\log ^{+} \log ^{+} \frac{1}{|g(0)|} . \\
\log ^{+} \frac{\left|g^{\prime \prime}(z)\right|}{|g(z)|} \leqq & C\left\{1+\log ^{+} \rho+\log ^{+} \frac{1}{\rho-\gamma}+\log ^{+} \mathfrak{R}+\log ^{+} \frac{1}{\delta(z)}\right. \\
& \left.+\log ^{+} \frac{1}{r}+\log ^{+} T(\rho, g)+\log ^{+} \log ^{+} \frac{1}{|g(0)|}\right\},
\end{aligned}
$$

where $\Re=n(\rho, g)+n(\rho, 1 / g), \delta(z)=\min \{|z-a|\}$, here a takes all zeros and poles of $g(z)$ in $|z| \leqq \rho$, where $C$ is an absolute constant.

Proof. For (2.5), see [4 Lemma 6.2]. Using Poisson-Jensen formula, we have for $|z| \leqq r<R$

$$
\begin{aligned}
\log g(z)= & \frac{1}{2 \pi} \int_{0}^{2 \pi} \log \left|g\left(\rho e^{\imath \phi}\right)\right| \frac{\rho e^{\imath \phi}+z}{\rho e^{\imath \phi}-z} d \phi \\
& -\sum_{j} \log \frac{\rho^{2}-\bar{a}_{j} z}{\rho\left(z-a_{j}\right)}+\sum_{k} \log \frac{\rho^{2}-b_{k} z}{\rho\left(z-b_{k}\right)}+i c,
\end{aligned}
$$

where $a_{\jmath}, b_{k}$ are zeros and poles of $g(z)$ in $|z| \leqq \rho$ respectively.

Differentiating this twice we have

$$
\begin{aligned}
\frac{g^{\prime \prime}(z)}{g(z)}-\left[\frac{g^{\prime}(z)}{g(z)}\right]^{2}= & \frac{1}{2 \pi} \int_{0}^{2 \pi} \log \left|g\left(\rho e^{\imath \phi}\right)\right| \frac{4 \rho e^{\imath \phi}}{\left(\rho e^{2 \phi}-z\right)^{3}} d \phi \\
& +\sum_{j}\left[\frac{\bar{a}_{j}^{2}}{\left.\rho^{2}-\bar{a}_{j} z\right)^{2}}-\frac{1}{\left(a_{j}-z\right)^{2}}\right]-\sum_{k}\left[\frac{\bar{b}_{k}^{2}}{\left(\rho^{2}-\bar{b}_{k} z\right)^{2}}-\frac{1}{\left(b_{k}-z\right)^{2}}\right] .
\end{aligned}
$$

Since $\left|z-a_{j}\right| \geqq \delta(z),\left|z-b_{k}\right| \geqq \delta(z)$, we have 


$$
\left|\frac{g^{\prime \prime}(z)}{g(z)}\right| \leqq\left|\frac{g^{\prime}(z)}{g(z)}\right|^{2}+\frac{4 \rho}{(\rho-r)^{3}}\left\{m(\rho, g)+m\left(\rho, \frac{1}{g}\right)\right\}+\Re\left[\frac{1}{(\rho-r)^{2}}+\frac{1}{\delta(z)^{2}}\right],
$$

Using (2.5), we prove (2.6).

\section{Proof of Lemma 1.}

Since Lemma A and D imply $P=\mu(2.3)$, we have

$$
\begin{aligned}
& T\left(\sigma r, f^{\prime \prime}\right)=\sigma^{\mu} T\left(r, f^{\prime \prime}\right)(1+o(1)), \quad 1<\sigma \leqq 36 . \\
& T\left(r, f^{\prime \prime}\right)=(1+o(1)) \frac{C(r) r^{\mu}}{\pi} \\
& |C(\sigma r)-C(r)|=o\{|C(r)|\} .
\end{aligned}
$$

Let $a_{l \nu}=\left\{l=1,2, \cdots, \tau ; \nu=1,2, \cdots, n_{l}\left(\alpha^{j+(3 / 2)}, f=a_{l}\right)\right\}$ be zeros of $f(z)-a_{l}$ in $|z| \leqq \alpha^{j+(3 / 2)}, \quad a_{l \nu}\left\{l=\tau+1, \cdots, \tau^{\prime} ; \nu=1,2, \cdots, n_{l}=n\left(\alpha^{j+(3 / 2)}, f^{\prime}=a_{l}\right)\right\}$ be zeros of $f^{\prime}(z)-a_{l}$ in $|z| \leqq \alpha^{j+(3 / 2)}$. Using Broutroux-Cartan's Theorem, the inequalities we have

$$
\begin{array}{ll}
\prod_{\nu=1}^{n l}\left|z-a_{l_{\nu}}\right|>\left(\frac{H}{\tau}\right)^{n_{l}}, & l=1,2, \cdots, \tau . \\
\prod_{\nu=1}^{n l}\left|z-a_{l \nu}\right|>\left(\frac{H}{\tau^{\prime}-\tau}\right)^{n_{l}} & l=\tau+1, \tau+2, \cdots, \tau^{\prime} .
\end{array}
$$

hold outside a finite number of small circles $\left(r_{j}^{1}\right),\left(r_{j}^{2}\right)$ respectively, and the sums of radii are no more than $\frac{2 e H}{\tau}, \frac{2 e H}{\tau^{\prime}-\tau}$ respectively. Set $\left(r_{j}^{3}\right)=\left\{\bigcup_{l=1}^{\tau^{\prime}} \bigcup_{\nu=1}^{n_{l}}\left(\left|z-a_{l_{\nu}}\right|\right.\right.$

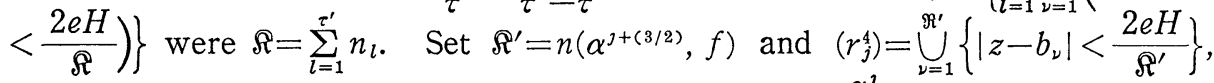
where $\left\{b_{\nu}\right\}$ is poles of $f(z)$ in $|z| \leqq \alpha^{j+(3 / 2)}$. Take $H=\frac{\alpha^{\jmath}}{54 e}(\alpha-1)$ and in Lemma $\mathrm{B}, \delta<\frac{\alpha-1}{36 e \alpha^{2}}$, and set $\left(r_{j}\right)=\bigcup_{i=1}^{4}\left(r_{j}^{i}\right) \cup E_{\jmath}$. The sum of diameters of $\left(r_{j}\right)$ is no more than $\frac{5}{9}(\alpha-1) \alpha^{j}$, but the width of $\left(\alpha^{\jmath}, \alpha^{\jmath+1}\right)$ is $(\alpha-1) \alpha^{j}$, hence there exists $\rho_{\jmath}, \rho_{j} \in\left(\alpha^{\jmath}, \alpha^{\jmath+1}\right)$ so that $\left(|z|=\rho_{j}\right) \cap\left(r_{j}\right)=\phi$. Set

$$
\delta_{0}=\min \left\{\delta\left(a_{1}, f\right), \cdots, \delta\left(a_{\tau}, f\right) ; \delta\left(a_{\tau+1}, f^{\prime}\right), \cdots, \delta\left(a_{\tau^{\prime}}, f^{\prime}\right)\right\} .
$$

Using Poisson-Jensen's formula, we have for $z=\rho_{j} e^{\imath \phi}$, from (3.2)

$$
\begin{aligned}
\log \frac{1}{\left|f(z)-a_{l}\right|} \leqq & \frac{\alpha^{\jmath+(3 / 2)}+\alpha^{\jmath+1}}{\alpha^{\jmath+(3 / 2)}-\alpha^{j+1}} \int_{0}^{2 \pi} \log ^{+} \frac{1}{\left|f\left(\alpha^{j+(3 / 2)} e^{i \theta}\right)-a_{l}\right|} d \theta \\
& +\sum_{\nu=1}^{n_{l}} \log \left|\frac{\left(\alpha^{\imath+3 / 2}\right)^{2}-\bar{a}_{l \nu} z}{\alpha^{j+(3 / 2)}\left(z-a_{l \nu}\right)}\right|
\end{aligned}
$$




$$
\begin{aligned}
& \leqq C m\left(\alpha^{j+3 / 2}, \frac{1}{f-a_{l}}\right)+\sum_{\nu=1}^{n_{l}} \log \frac{2 \alpha^{j+3 / 2}}{\left|z-a_{l \nu}\right|} \\
& \leqq C m\left(\alpha^{j+3 / 2}, \frac{1}{f-a_{l}}\right)+n\left(\alpha^{j+3 / 2}, f=a_{l}\right) \log \frac{2 \tau \alpha^{j+3 / 2}}{H} \\
& \leqq C T\left(\alpha^{j+2}, \frac{1}{f-a_{l}}\right) \leqq C T\left(\alpha^{j+2}, f\right) .
\end{aligned}
$$

By Lemma C,

$$
T\left(\alpha^{\jmath+2}, f\right) \leqq C\left\{T\left(2 \alpha^{\jmath+2}, f^{\prime}\right)\right\} \leqq C\left\{T\left(4 \alpha^{\jmath+2}, f^{\prime \prime}\right)\right\} .
$$

Using (3.1) we have

$$
T\left(4 \alpha^{\jmath+2}, f^{\prime \prime}\right)<2\left(4 \alpha^{2}\right)^{\mu} T\left(\alpha^{\jmath}, f^{\prime \prime}\right)<2\left(4 \alpha^{2}\right)^{\mu} T\left(\rho_{\jmath}, f^{\prime \prime}\right) .
$$

Since $\sum_{a} \delta\left(a, f^{\prime}\right)=2$, the order of $f^{\prime}(z)$ equals lower order $\mu(2.3)$ and the order of $f(z)$ equals $\mu<\infty$. So that

$$
\begin{aligned}
T\left(r, f^{\prime \prime}\right) & =m\left(r, f^{\prime \prime}\right)+N\left(r, f^{\prime \prime}\right) \\
& \leqq m\left(r, \frac{f^{\prime \prime}}{f}\right)+m(r, f)+3 N(r, f) \leqq 4 T(r, f) .
\end{aligned}
$$

Using (3.5)-(3.8), we have

$$
\log \frac{1}{\left|f\left(\rho_{j} e^{2 \phi}\right)-a_{l}\right|} \leqq C T\left(\rho_{\jmath}, f\right)
$$

where $C$ is constant that is not depedent on $j, l$. by (3.9)

For $l=1,2, \cdots, \tau$, set $E_{l_{\jmath}}^{*}=E\left\{\phi ; o \leqq \phi<2 \pi, \log \frac{1}{\left|f\left(\rho_{j} e^{\imath \phi}\right)-a_{l}\right|}>\frac{1}{2} m\left(\rho_{\jmath}, \frac{1}{f-a_{l}}\right)\right\}$

$$
\begin{aligned}
m\left(\rho_{\jmath}, \frac{1}{f-a_{l}}\right) & =\frac{1}{2 \pi}\left[\int_{E_{l \jmath}^{*}}+\int_{C E_{l \jmath}^{*}}\right] \log ^{+} \frac{1}{\left|f\left(\rho_{j} e^{\imath \phi}\right)-a_{l}\right|} d \phi \\
& \leqq \frac{C}{2 \pi} T\left(\rho_{\jmath}, f\right) \text { meas } E_{l j}^{*}+\frac{1}{2} m\left(\rho_{\jmath}, \frac{1}{f-a_{l}}\right)
\end{aligned}
$$

so that

$$
\text { meas } E_{l \jmath}^{*} \leqq \frac{\pi}{C} \frac{m\left(\rho_{\jmath}, \frac{1}{f-a_{l}}\right)}{T\left(\rho_{\jmath}, f\right)} \geqq \frac{\pi}{2 C} \delta\left(a_{l}, f\right) \geqq \frac{\pi}{2 C} \delta_{0}, \quad\left(j \geqq j_{0}\right) .
$$

Hence for $z \in E_{l j}=\left\{z ; z=\rho_{j} e^{\imath \phi}, \phi \in E_{l_{j}}^{*}\right\}$, using (3.8) we have

$$
\log \frac{1}{\left|f\left(\rho_{j} e^{\imath \phi}\right)-a_{l}\right|}>\frac{1}{2} m\left(\rho_{\jmath}, \frac{1}{f-a_{l}}\right)>\frac{\delta_{0}}{4} T\left(\rho_{\jmath}, f\right)>\frac{\delta_{0}}{16} T\left(\rho_{\jmath}, f^{\prime \prime}\right) .
$$

By Lemma 2, set $g(z)=f(z)-a_{l}, \rho=\alpha^{j+(3 / 2)}, r=\rho_{\jmath}$, for $z \in E_{l \jmath}(l=1,2, \cdots, \tau)$ 


$$
\begin{aligned}
& \log ^{+} \frac{\left|f^{\prime}(z)\right|}{\left|f(z)-a_{l}\right|} \leqq C\left\{1+\log ^{+} \alpha^{\jmath+3 / 2}+3 \log ^{+} \frac{1}{\alpha^{\jmath+3 / 2}-\rho_{\jmath}}\right. \\
& +\log ^{+}\left(n_{l}+\Re^{\prime}\right)+\log ^{+} \frac{1}{\delta(z)}+\log ^{+} T\left(\alpha^{j+3 / 2}, f-a_{l}\right) \\
& \left.+\log ^{+} \log ^{+} \frac{1}{\left|f(0)-a_{l}\right|}\right\}
\end{aligned}
$$

From (3.6)-(3.8), $l=1,2, \cdots, \tau$, we have

$$
\begin{aligned}
& n_{l}=n\left(\alpha^{\jmath+3 / 2}, \frac{1}{f-a_{l}}\right) \leqq \frac{2}{\log \alpha} N\left(\alpha^{\jmath+2}, \frac{1}{f-a_{l}}\right) \leqq C T\left(\rho_{\jmath}, f\right) . \\
& \mathfrak{R}^{\prime}=n\left(\alpha^{\jmath+3 / 2}, f-a_{l}\right)=n\left(\alpha^{j+3 / 2}, f\right) \leqq C T\left(\rho_{\jmath}, f\right) .
\end{aligned}
$$

and for $l=\tau+1, \cdots, \tau^{\prime}$, by the same reasoning we have

so

$$
n_{l}=n\left(\alpha^{\jmath+3 / 2}, \frac{1}{f^{\prime}-a}\right)<C T\left(\rho_{\jmath}, f\right) \text {. }
$$

$$
\Re=\sum_{l=1}^{\tau^{\prime}} n_{l}<C T\left(\rho_{\jmath}, f\right), \quad n_{l}+\mathscr{R}^{\prime} \leqq C T\left(\rho_{\jmath}, f\right) .
$$

From definition of $\left(r_{j}^{3}\right),\left(r_{j}^{4}\right)$, using (3.13) we have

$$
\delta(z) \geqq \frac{2 e H}{\mathfrak{R}+\mathbb{R}^{\prime}}>\frac{C \alpha^{\jmath}}{T\left(\rho_{\jmath}, f\right)} .
$$

Combing (3.12)-(3.14), for $z \in E_{l \jmath}$, we have

$$
\log \frac{\left|f^{\prime}(z)\right|}{\left|f(z)-a_{l}\right|}<C\left\{1+\log \rho_{j}+\log T\left(\rho_{\jmath}, f\right)\right\} .
$$

Hence from (3.11), (3.6), (3.7) for $z \in E_{l \jmath}, l=1,2, \cdots, \tau, r>r_{0}$ we have

$$
\begin{aligned}
& \log \left|f^{\prime}(z)\right| \leqq \log ^{+} \frac{\left|f^{\prime}(z)\right|}{\left|f(z)-a_{l}\right|}+\log \left|f(z)-a_{l}\right| \\
& =\log ^{+} \frac{\left|f^{\prime}(z)\right|}{\left|f(z)-a_{l}\right|}-\log \frac{1}{\left|f(z)-a_{l}\right|} \\
& \leqq C\left\{1+\log \rho_{j}+\log T\left(\rho_{\jmath}, f\right)\right\}-\frac{\delta_{0}}{16} T\left(\rho_{\jmath}, f^{\prime \prime}\right) \\
& \leqq C\left\{1+\log \rho_{j}+\log T\left(\rho_{\jmath}, f^{\prime \prime}\right)\right\}-\frac{\delta_{0}}{16} T\left(\rho_{\jmath}, f^{\prime \prime}\right) \\
& <-\frac{\delta_{0}}{64} T\left(\rho_{\jmath}, f^{\prime \prime}\right) \text {. } \\
& \log \left|f^{\prime \prime}(z)\right|<-\frac{\delta_{0}}{64} T\left(\rho_{\jmath}, f^{\prime \prime}\right) \text {. }
\end{aligned}
$$


For $\tau<l \leqq \tau^{\prime}$, set $E_{l j}^{*}=\left\{\phi ; 0 \leqq \phi<2 \pi, \log \frac{1}{\left|f^{\prime}\left(\rho_{j} e^{\imath \phi}\right)-a_{l}\right|}>\frac{1}{2} m\left(\rho_{\jmath}, \frac{1}{f-a_{l}}\right)\right\}$ and $E_{l j}=\left\{z ; z=\rho_{j} e^{i \phi}, \phi \in E_{l j}^{*}\right\}$, with the same reasoning we can prove $l=\tau+1, \cdots, \tau^{\prime}$, $z \in E_{l,}$

$$
\begin{aligned}
& \log \frac{1}{\left|f^{\prime}\left(\rho_{j} e^{2 \phi}\right)-a_{l}\right|}>\frac{\delta_{0}}{16} T\left(\rho_{\jmath}, f^{\prime \prime}\right), \\
& \log \left|f^{\prime \prime}(z)\right|<-\frac{\delta_{0}}{64} T\left(\rho_{\jmath}, f^{\prime \prime}\right) .
\end{aligned}
$$

Combining (3.11), (3.15)-(3.18), we can prove Lemma 1.

\section{Proof of Theorem.}

Set $C\left(\alpha^{j}\right)=\left|C\left(\alpha^{j}\right)\right| e^{\imath \omega_{j}}$. We divide $|z|=\rho_{\text {j }}$ into $2 \mu \operatorname{arcs} \alpha_{1}, \cdots, \alpha_{\mu} ; \beta_{1}, \cdots, \beta_{\mu}$, so that $\cos \left(\mu \phi+\omega_{j}\right) \geqq 0$ on $\alpha_{k}(k=1,2, \cdots, \mu) ; \cos \left(\mu \phi+\omega_{j}\right) \leqq 0$ on $\beta_{k}(k=1,2, \cdots$, $\mu)$. Also set

$$
\begin{aligned}
& \alpha_{k}(\phi)=\left\{\phi ; 0 \leqq \phi<2 \pi, \rho_{j} e^{\imath \phi} \in \alpha_{k}\right\}=\left\{\phi_{j k}, \phi_{j k}+\frac{\pi}{\mu}\right\} \\
& \beta_{k}(\phi)=\left\{\phi ; 0 \leqq \phi<2 \pi, \rho_{j} e^{\imath \phi} \in \beta_{k}\right\}=\left\{\Psi_{j k}, \Psi_{j k}+\frac{\pi}{\mu}\right\} .
\end{aligned}
$$

And $\phi_{0}<\frac{1}{10 \mu} \eta$, we set

$$
\bar{\alpha}_{k}(\phi)=\left\{\phi_{j k}+\phi_{0}, \phi_{j k}+\frac{\pi}{\mu}-\phi_{0}\right\} ; \quad \tilde{\beta}_{k}(\phi)=\left\{\Psi_{j k}+\phi_{0}, \Psi_{j k}+\frac{\pi}{\mu}-\phi_{0}\right\} .
$$

By Lemma B, for $z \in \tilde{\alpha}_{k}=\left\{z ; z=\rho_{j} e^{i \phi}, \phi \in \hat{\alpha}_{k}(\phi)\right\}$,

$$
\log \left|f^{\prime \prime}\left(\rho_{j} e^{\imath \phi}\right)\right| \geqq \operatorname{Re}\left\{C_{j} z^{\mu}\right\}-4 \varepsilon\left|C_{j}\right| \rho_{j}^{\mu},
$$

where $\varepsilon<\frac{\sin \mu \phi_{0}}{10}$, for $z \in \tilde{\alpha}_{k}$

$$
\begin{aligned}
\log \left|f^{\prime \prime}\left(\rho_{j} e^{\imath \phi}\right)\right| & \geqq\left|C_{j}\right| \rho_{j}^{\mu} \cos \left(\mu \phi+\omega_{j}\right)-4 \varepsilon \rho_{j}^{\mu}\left|C_{j}\right| \\
& \geqq\left|C_{j}\right| \rho_{j}^{\mu} \sin \mu \phi_{0}-4 \varepsilon \rho_{j}^{\mu}\left|C_{j}\right| \\
& >\frac{1}{2}\left|C_{j}\right| \rho_{j}^{\mu} \sin \mu \phi_{0}(>0) .
\end{aligned}
$$

Assume that $\left\{a_{1}, a_{2}, \cdots, a_{\tau}\right\},\left\{a_{\tau+1}, \cdots, a_{\tau^{\prime}}\right\}$ are arbitrarily taken as finite deficients of $f(z)$ and $f^{\prime}(z)$ respectively and $\left\{\rho_{j}\right\}, E_{l \jmath}\left(l=1,2, \cdots, \tau^{\prime}\right)$ are the same as Lemma 1. Using Lemma 1 and (4.1) we have $\left(\bigcup_{l=1}^{\tau^{\prime}} E_{l \jmath}\right) \cap\left(\bigcup_{k=1}^{\mu} \tilde{\alpha}_{k}\right)=\phi$. Because from Lemma 1 meas $E_{l_{\jmath}}^{*}>\eta\left(l=1,2, \cdots, \tau^{\prime}\right)$ and $2 \mu \phi_{0}<\eta / 5$, so we have a $\tilde{\beta}_{l^{\prime}}, E_{l_{\jmath}}$ $\cap \tilde{\beta}_{l^{\prime}} \neq \phi\left(l=1,2, \cdots, \tau, \tau+1, \cdots, \tau^{\prime} ; l^{\prime} \in(1,2, \cdots \mu)\right)$. From Lemma B, $z \in \tilde{\beta}_{l}$, 


$$
\begin{aligned}
\log \left|f^{\prime \prime}(z)\right| & \leqq \operatorname{Re}\left\{C_{j} z^{\mu}\right\}+4 \varepsilon\left|C_{j}\right| \rho_{j}^{\mu} \\
& =\left|C_{j}\right| \rho_{j}^{\mu} \cos \left(\mu \phi+\omega_{j}\right)+4 \varepsilon\left|C_{j}\right| \rho_{j}^{\mu} \\
& \leqq-\rho_{j}^{\mu}\left|C_{j}\right| \sin \left(\mu \phi_{0}\right)+4 \varepsilon\left|C_{j}\right| \rho_{j}^{\mu} \leqq-\frac{1}{2} \sin \left(\mu \phi_{0}\right)\left|C_{j}\right| \rho_{j}^{\mu} .
\end{aligned}
$$

From (3.1) and $\mu=P$, we have

$$
\begin{gathered}
\left|C_{j}\right| \rho_{j}^{\mu}>\left|C_{j}\right|\left(\alpha^{j}\right)^{\mu}>\frac{\pi}{2} T\left(\alpha^{\jmath}, f^{\prime \prime}\right)>\frac{\pi}{4}\left(\frac{\alpha^{2}}{\rho_{\jmath}}\right)^{\mu} T\left(\rho_{\jmath}, f^{\prime \prime}\right) \\
>\frac{\pi}{4}\left(\frac{\alpha^{\jmath}}{\alpha^{\jmath+1}}\right)^{\mu} T\left(\rho_{\jmath}, f^{\prime \prime}\right)=\frac{\pi}{4} \alpha^{-\mu} T\left(\rho_{\jmath}, f^{\prime \prime}\right) .
\end{gathered}
$$

Therefore, from (4.2) there exists a positive $C^{\prime}$ that is independent of 3 such that for $z \in \tilde{\beta}_{l}$,

$$
\log \left|f^{\prime \prime}(z)\right| \leqq-C^{\prime} T\left(\rho_{\jmath}, f^{\prime \prime}\right) .
$$

Supose $z_{0} \in E_{l j} \cap \tilde{\beta}_{l^{\prime}}$ for $z \in \tilde{\beta}_{l^{\prime}}$, from (4.3), (2.1) we have

$$
\begin{aligned}
\left|f^{\prime}(z)\right| & \leqq \rho_{j} \int_{\overparen{z_{0} z}}\left|f^{\prime \prime}(z)\right| d \phi+\left|f^{\prime}\left(z_{0}\right)\right| \\
& \leqq 2 \pi \rho_{j} e^{-C^{\prime} T\left(\rho_{j}, f^{\prime \prime}\right)}+e^{-k T\left(\rho_{j}, f^{\prime \prime}\right)} \leqq e^{-C T\left(\rho_{\rho}, f^{\prime \prime}\right)} .
\end{aligned}
$$

were $\widetilde{z_{0} z} \subset \tilde{\beta}_{l^{\prime}}, C$ independent of $\jmath$. Therfore we have for $z \in \tilde{\beta}_{l^{\prime}}, l=1,2, \cdots, \tau$,

$$
\begin{aligned}
\left|f(z)-a_{l}\right| & \leqq\left|f(z)-f\left(z_{0}\right)\right|+\left|f\left(z_{0}\right)-a_{l}\right| \leqq \rho_{j} \int_{\widehat{z_{0}}}\left|f^{\prime}(z)\right| d \phi+e^{-k T\left(\rho_{\rho}, f^{\prime \prime}\right)} \\
& \leqq 2 \pi \rho_{j} e^{-C^{\prime} T\left(\rho_{\rho}, f^{\prime \prime}\right)}+e^{-k T\left(\rho_{\rho}, f^{\prime \prime}\right)} \leqq e^{-C T\left(\rho_{f}, f^{\prime \prime}\right)}
\end{aligned}
$$

From the same reason for $z \in \tilde{\beta}_{l^{\prime}}, l=\tau+1, \cdots, \tau^{\prime}$,

$$
\left|f^{\prime}(z)-a_{l}\right|<e^{-C T\left(\rho_{j}, f^{\prime \prime}\right)} \text {. }
$$

$a_{l_{1}} \neq a_{l_{2}}$ for $1 \leqq l_{1}<l_{2} \leqq \tau$ implies $\tilde{\beta}_{l_{1}^{\prime}} \neq \tilde{\beta}_{l_{2}^{\prime}}$. In fact, if $\beta_{l_{1}^{\prime}}=\beta_{l_{2}^{\prime}}$ then we have from (4.5)

$$
\left|a_{l_{1}}-a_{l_{2}}\right| \leqq\left|f(z)-a_{l}\right|+\left|f(z)-a_{l_{2}}\right| \leqq 2 e^{-C T\left(\rho_{2}, f^{\prime \prime}\right)} .
$$

Setting $j \rightarrow \infty$, we have $a_{l_{1}}=a_{l_{2}}$. This is a contradiction. By the same reasoning $\tilde{\beta}_{l_{1}} \neq \tilde{\beta}_{l_{2}}\left(\tau+1 \leqq l_{1}<l_{2}<\tau^{\prime}\right)$. And from (4.4) and (4.6) $\tilde{\beta}_{l_{1}} \neq \tilde{\beta}_{l_{2}}\left\{l_{1}=1,2, \cdots, \tau\right.$; $l_{2}=\tau+1, \cdots, \tau^{\prime}$; and $\left.a_{l} \neq 0, l \in\left(\tau+1, \cdots, \tau^{\prime}\right)\right\}$. Because the number of $\tilde{\beta}_{l}$ is at most $\mu$, so the Theorem has been proved.

From the proof we have following Corollary,

Corollary. Let $f(z)$ be a meromorphic function of lower order $\mu<\infty$, if

$$
\sum_{a} \delta\left(a, f^{\prime}\right)=2,
$$

then the number of deficient values of $f^{\prime}(z) \nu\left(f^{\prime}\right) \leqq \mu+1$. 


\section{REFERENCES}

[1] EDREI, A. AND Fuchs, W. H. J., Valeurs deficientes et valeurs asymptotiques des fonctions meromorphes, Comment. Math. Helv., 33 (1959), 258-295.

[2] EDREI, A. AND FUCHS, W. H. J., On the growth of meromorphic functions with several deficient values, Trans. Amer. Math. Soc., 93 (1959), 293-328.

[3] Ozawa, M., On the deficiencies of meromorphic functions, Kodai. Math. Sem. Rep., 20 (1968), 385-388.

[4] YANG LE, Value Distribution and it's new research, Beijing. 1982.

Department of Mathematics

EAst China Normal University

Shanghai, P.R. China 